\title{
Tendencias Pedagógicas y Herramientas Digitales en el Aula
}

\section{Pedagogical Trends and Digital Tools in the Classroom}

\author{
Dany Cristian López-Espinoza \\ dalo.7@hotmail.com \\ Universidad Católica Santiago de Guayaquil, Guayaquil \\ Ecuador \\ https://orcid.org/0000-0002-6951-0696 \\ Ángel Enrique Azuero-Azuero \\ Universidad Católica de Cuenca, Cuenca \\ angelazuero@hotmail.com \\ Ecuador \\ https://orcid.org/0000-0003-2176-597X
}

Recibido: 15 de agosto de 2019

Aprobado: 15 de septiembre de 2019

\section{RESUMEN}

La finalidad de esta investigación fue estudiar la Tendencias Pedagógicas y Herramientas Digitales en el Aula: Una Propuesta para su Inclusión Adecuada, en la Carrera de Contabilidad y Auditoría de la Universidad Católica De Cuenca, Extensión La Troncal; cuya razón principal es dar a conocer la importancia que tienen dentro del contexto educativo .Se desarrolló desde un enfoque mixto; bajo un diseño no experimental transeccional; se utilizó la recolección de datos para establecer patrones de comportamiento, con base en la medición numérica y el análisis estadístico; en cuanto a la parte cualitativa se utilizó la recolección de datos sin medición numérica para descubrir o afinar preguntas de investigación en el proceso de interpretación. Concluyendo así que entre lo más importante se encuentra el fortalecimiento y la actualización del personal docente en cuanto a la práctica de nuevas tendencias pedagógicas y el uso adecuado de las herramientas digitales en el aula.

Descriptores: tendencias pedagógicas; herramientas digitales; inclusión adecuada; educación superior; investigación mixta. 


\author{
CIENCIAMATRIA \\ Revista Interdisciplinaria de Humanidades, Educación, Ciencia y Tecnología \\ Año VI. Vol. VI. N¹. Edición Especial. 2020 \\ Hecho el depósito de ley: pp201602FA4721 \\ ISSN-L: 2542-3029; ISSN: 2610-802X \\ Universidad Nacional Experimental Francisco de Miranda (UNEFM). Santa Ana de Coro. Venezuela \\ Dany Cristian López-Espinoza; Ángel Enrique Azuero-Azuero
}

\begin{abstract}
The purpose of this research was to study the Pedagogical Trends and Digital Tools in the Classroom: A Proposal for Adequate Inclusion, in the Accounting and Audit Career of the Catholic University of Cuenca, La Troncal Extension; It was developed from a mixed approach; data collection was used to establish patterns of behavior, based on numerical measurement and statistical analysis; As regards the qualitative part, data collection without numerical measurement was used to discover or refine research questions in the interpretation process. Thus concluding that it is important to strengthen and update the teaching staff regarding the practice of new pedagogical trends and the proper use of digital tools in the classroom.
\end{abstract}

Descriptors: pedagogical trends; digital tools; adequate inclusion; higher education; mixed research.

\title{
INTRODUCCIÓN
}

La educación en el Ecuador, atraviesa una evolución profunda en busca de la excelencia e innovación académica. Las Tecnologías de la Información y Comunicación (TIC) constituyen un factor preponderante de dicho cambio, en este sentido, la Ley Orgánica de Educación Superior (LOES, 2010) vigente en el país, establece que son derechos de las y los estudiantes contar y acceder a los medios y recursos adecuados para su formación superior y que están garantizados por la Constitución. La educación superior tiene como fines, aportar al desarrollo del pensamiento universal, la expansión de la elaboración científica y al desarrollo de las transmisiones y creaciones tecnológicas, para ello, los órganos educativos superiores aplican necesariamente la práctica de operativos informáticos con software libre.

Incluir herramientas digitales en el aula, demanda del docente un dominio en el empleo de un modelo educativo virtual, cuyo objetivo es la comunicación educativa apoyada en el uso pedagógico de las TIC. Evidentemente, la tecnología móvil y otros referentes tecnológicos tienden a crecer potencialmente a nivel global, lo cual hace que se encuentren inmersos en el ámbito educativo en todos los niveles y por ello se vuelve necesario una atención especial por parte de los educadores. La sociedad educativa de hoy, específicamente en los estudiantes, demandan innovadoras formas de enseñar por parte de los docentes, de los cuales ya no esperan la catedra 
Dany Cristian López-Espinoza; Ángel Enrique Azuero-Azuero

tradicional sino más bien tienen la expectativa de algo nuevo a la hora de aprender, que los motive a favorecer su conocimiento.

Dado el caso que el uso de herramientas digitales en el aula no se limita a la mera utilización del recurso como tal, sino que ha de ser aprovechado de una forma pedagógica, es decir, con el claro propósito de facilitar el aprendizaje, es necesario brindar a los profesores universitarios un asesoramiento oportuno, claro y preciso de cuáles son y del cómo utilizar las TIC disponibles, pero con orientaciones educativas adecuadas, es decir, darles a conocer las alternativas pedagógicas y tecnológicas que están en auge y que tienen disponibilidad, con ello se podrá evitar que el proceso educativo se vea limitado a cátedras tradicionales sujetas a una simple presentación de diapositivas, procesador de texto u otros métodos elementales de enseñanza.

En definitiva, en la variedad de alternativas tecnológicas existentes y útiles en el campo educativo formal se puede evidenciar que la lista es extensa y quizá interminable, pero hay que insistir en las competencias de los docentes de educación superior al respecto, con especial atención en el contexto investigado, pues, como afirman Hernández, Ayala-García y Gamboa (2015), aparentemente "no existen estándares ni se ha desarrollado un modelo que describa y evalué las competencias TIC de los docentes de educación superior" (p. 231), siendo pertinente contar con este perfil, por cuanto las TIC son necesarias en la educación presente y futura tal como establece Lattá Arias (2019).

Por todo lo que antecede al respecto de las tendencias pedagógicas y las herramientas digitales en el aula, se puede deducir, que los docentes universitarios se encuentran actualmente atravesando un proceso de transición hacia un nuevo modelo de enseñanza aprendizaje el cual tiene una rápida evolución y emerge la necesidad de la denominada alfabetización tecnológica.

\section{DESARROLLO TEÓRICO}

Los sistemas educativos y con mayor relevancia el sistema de educación superior, está llamado a cubrir las demandas y expectativas de una trasformación social que se está viviendo en el mundo gracias a los avances científicos y tecnológicos. Entre 
Dany Cristian López-Espinoza; Ángel Enrique Azuero-Azuero

las ventajas que han provocado las herramientas digitales, está la posibilidad de una comunicación instantánea y el intercambio de conocimientos. Todo esto ha dado paso a la internacionalización de las universidades.

En este sentido, cada vez más la educación superior, a nivel mundial, tiene que responder a tendencias macro que la están afectando como el movimiento hacia una sociedad y economía del conocimiento; el desarrollo en tecnología informática y comunicaciones; la mayor movilidad de la gente, capital, ideas, conocimiento y tecnología; el mayor énfasis en la economía de mercado; los cambios en la gobernabilidad, desde lo nacional a regional e internacional; la privatización de la educación, así como también la importancia cada vez mayor de un aprendizaje permanente para toda la vida. (Manzanilla et al 2016, p. 8)

\section{Las TIC y su papel protagónico.}

En este contexto el uso efectivo de las TIC cumple un papel protagónico y la formación-preparación en ellas augura una mejor posibilidad de vida, de recortar la brecha digital entre los países más atrasados, además de servir como motor de desarrollo económico y de ascenso en el nivel social (Manzanilla et al., 2016). Además, su incorporación en la cultura universitaria necesita de ciertos prerrequisitos: la visión estratégica, la capacidad de aprender y adoptar buenas prácticas a través del benchmarking (proceso sistemático y continuo para evaluar los productos), el currículum de formación y el modelo educativo que le da sustentación teórica a las propuestas de desarrollo docente asociada a la inclusión de las TIC en las prácticas de enseñanza aprendizaje. (Fainholc, Nervi, Romero y Halal, 2013, p. 3)

Es el profesor bajo una visión de facilitador del aprendizaje y no simplemente de transmisor de conocimientos, quien asume la responsabilidad ética y profesional de aprovechar los beneficios de las herramientas digitales a su alcance, como un elemento clave para lograr la internacionalización de la educación superior. Se podría precisar que la tecnología por sí sola no produce aprendizaje significativo y es la intervención docente, a través de las tendencias pedagógicas, lo que garantizará esta producción. 
Dany Cristian López-Espinoza; Ángel Enrique Azuero-Azuero

Un líder pedagógico es aquel docente que centra su quehacer profesional en mejorar sus propias prácticas de enseñanza, es quién se enfoca en los aspectos relacionados al proceso de enseñanza y aprendizaje para favorecer y potenciar de una forma más innovadora la adquisición de nuevos conocimientos por parte del estudiantado (Sandí y Cruz, 2016).

El docente es un formador que centra su labor en el estudiante, desafío que le exige la ejecución de actividades de aprendizaje que promueva el desarrollo del trabajo autónomo y reflexivo; en este sentido las oportunidades de promover prácticas de integración de las TIC como elemento innovador en el contexto de las actividades de aprendizaje puede resultar beneficiosa ante las necesidades de aprender mejor de los estudiantes, pero también amerita por parte del docente la adquisición de competencias digitales como: tecnológica, comunicativa, informacional, y de aprendizaje (Fainholc, et al., 2013).

Es así que usualmente, se asocia el uso de las TIC a la innovación, sin embargo, se debe tener en cuenta que el utilizar las TIC en el proceso de enseñanza y aprendizaje no necesariamente implica innovación, ya que la innovación radica en las estrategias didácticas que se configuren y desarrollen con las TIC como herramientas de apoyo y mediadoras en el proceso. (Sandí y Cruz, 2016, p. 5). Las estrategias didácticas a las que se hace mención, están implícitas en el conocimiento pedagógico que posean los catedráticos de la universidad y esto definirá la efectividad de un proceso de formación académica.

El concepto del conocimiento pedagógico del contenido (CPC) establece una aportación de especial utilidad y por esta razón se ha aplicado su estudio teórico y práctico. La fundamentación de este conocimiento está otorgada al estudio que sobrepasa el tema de la materia en sí misma y que alcanza la dimensión de la materia para la educación. EI CPC necesita algo por encima del conocimiento de la situación o nociones de un dominio, se necesita abordar las estructuras del tema.

En cambio, el conocimiento del contenido temático está enfocado a que educar, el CPC se dirige al cómo educar. Se incluyen en el CPC "las formas más útiles de representación de las ideas; las analogías, ilustraciones, ejemplos, explicaciones y 
Dany Cristian López-Espinoza; Ángel Enrique Azuero-Azuero

demostraciones más poderosos, en pocas palabras, las formas de representación y formulación del tema que lo hace comprensible a otros" (Vicedo, 2015, p. 424). Uno de los elementos que motivan esta investigación es la pedagogía como tal, aplicada en el contexto educativo a nivel superior y de sobremanera en el Ecuador.

La catedra del maestro universitario ecuatoriano, posiblemente se ha fundamentado principalmente en sus experiencias profesionales específicas y que quizá muy pocas veces han tenido un matiz pedagógico en el aula, lo cual de ninguna manera pone en tela de duda su capacidad profesional para desempeñar el rol del profesorado. Sin embargo, es demandante la necesidad de activar o potenciar los ambientes pedagógicos en las aulas universitarias y esto amerita el desempeño de un papel más activo del estudiante y del profesor.

Pérez, Betancourt, Silveira y Loza. (2016), mencionaron que la pedagogía o educación afectiva que necesita la Universidad Ecuatoriana y que debe poseer una perspectiva humanista y cognitiva, así también, concluyen que la educación es una de las categorías de la pedagogía, es una parte esencial de la vida, un componente de la práctica social. Esto invita a reflexionar acerca de algunos referentes a nivel internacional, sobre las tendencias pedagógicas que en la actualidad indiscutiblemente van acompañadas de herramientas digitales como recursos didácticos y que gracias a la conectividad existente en el planeta son accesibles, para que a su vez puedan ser adaptadas al sistema educativo nacional.

\section{Tendencias pedagógicas y tecnológicas, como producto de un ejercicio práctico basado en la metodología Delphi}

Aprendizaje Basado en Retos. Es una estrategia que proporciona a los estudiantes un contexto general en el que ellos, de manera colaborativa, deben determinar el reto a resolver. Los estudiantes trabajan con sus profesores y expertos para resolver este desafío en comunidades de todo el mundo y así desarrollar un conocimiento más profundo de los temas que estén estudiando.

Aprendizaje Basado en Proyectos. Técnica didáctica que se orienta al diseño y desarrollo de un proyecto de manera colaborativa por un grupo de alumnos. Lo anterior como una forma de lograr los objetivos de aprendizaje de una o más áreas 
Dany Cristian López-Espinoza; Ángel Enrique Azuero-Azuero

disciplinares y además, lograr el desarrollo de competencias relacionadas con la administración de proyectos reales, siendo pertinente contar con la investigación acción como método genérico en el aprendizaje por proyectos tal como plantean García Morales \& González Ten Meer (2018).

Aprendizaje Invertido. Es una técnica didáctica en la que la exposición de contenido se hace por medio de videos que pueden ser consultados en línea de manera libre, mientras el tiempo de aula se dedica a la discusión, resolución de problemas y actividades prácticas bajo la supervisión y asesoría del profesor.

Aprendizaje Vivencial. Es el patrón de aprendizaje que involucra la experiencia de una práctica en la que el educando puede realizar actividades que robustece sus aprendizajes.

Aprendizaje Flexible. Se enfoca en ofrecer opciones al estudiante del cuándo, dónde y cómo aprender. Esto puede ayudar a los estudiantes a cubrir sus necesidades particulares ya que tendrán mayor flexibilidad en el ritmo, lugar y forma de entrega de los contenidos educativos. El aprendizaje flexible puede incluir el uso de tecnología para el estudio en línea, dedicación a medio tiempo, aceleración o desaceleración de programas, entre otros. (pp. 10-13)

Esto, en cuanto a lo que se refiere algunas tendencias en pedagogía. Todas ellas han sido adoptadas en diferentes instituciones de educación superior, en donde se ha podido tener una experiencia satisfactoria en el desarrollo del conocimiento de los estudiantes. Cabe mencionar que están apoyadas en la tecnología para lograr su efectividad. (Observatorio de Innovación Educativa 2016).

\section{Tendencias en tecnología (herramientas digitales)}

Aprendizaje Adaptativo. Es un método de instrucción que utiliza un sistema computacional para crear una experiencia personalizada de aprendizaje. La instrucción, retroalimentación y corrección se ajusta con base en las interacciones del estudiante y al nivel desempeño demostrado.

Aprendizaje en Redes Sociales y Entornos Colaborativos. Se refiere al uso de plataformas existentes o propietarias, que potencializan el aprendizaje social y colaborativo independientemente de dónde se encuentren los participantes. Se vale 
Dany Cristian López-Espinoza; Ángel Enrique Azuero-Azuero

de diversos recursos tecnológicos como: redes sociales, blogs, chats, conferencias en línea, pizarra compartida, wikis, entre otros recursos, a menudo alojados en la nube.

Entornos Personalizados de Aprendizaje. Son sistemas que los estudiantes pueden configurar ellos mismos para tomar el control y gestión de su propio aprendizaje. Esto incluye el establecimiento de objetivos de aprendizaje, la gestión de los contenidos y comunicaciones con otros estudiantes. Estos entornos pueden estar compuestos de uno o varios subsistemas, por ejemplo, un sistema de gestión de aprendizaje LMS, blogs, feeds, etc. Puede tratarse de una aplicación de escritorio o bien estar compuestos por uno o más servicios web.

Insignias (badges) y Microcréditos. Las insignias son un mecanismo para otorgar certificación a los estudiantes de un aprendizaje informal en forma de microcréditos. Los estudiantes pueden recopilarlas, organizarlas y publicarlas para demostrar sus habilidades y logros, en diferentes sitios web, como redes sociales, redes profesionales, comunidades virtuales, entre otros.

Realidad Aumentada. Uso de tecnología que complementa la percepción e interacción con el mundo real y permite al estudiante sobreponer una capa de información a la realidad, proporcionando así experiencias de aprendizaje más ricas e inmersivas. (Observatorio de Innovación Educativa 2016 pp. 15-18)

La integración en el sistema de educación superior, de estas y otras herramientas digitales conocidas también como TIC, ha permitido el incremento de la oferta informativa, construcción de espacios dúctiles para la enseñanza, exclusión de los impedimentos de espacio y tiempo, aumento de las formas de comunicación, aumento de los espacios interactivos, beneficio sobre la enseñanza independiente y del aprendizaje individual, facilitar formación permanente, etc. (Morales, Trujillo y Raso, 2015, p. 105).

Se puede contrastar en numerosos estudios que una de las salvedades más importantes sobre la asimilación de las TIC en los espacios de enseñanza es la 
Dany Cristian López-Espinoza; Ángel Enrique Azuero-Azuero

instrucción y adaptación de los docentes. Un criterio importante ante este aspecto es la facilidad de cambio permanente de las tecnologías, con lo que implica una formación continua de los docentes ante esta situación. (Morales et al., 2015, p. 105).

Es probable que los estudiantes universitarios, hayan adquirido habilidades expertas en el manejo de algunos recursos tecnológicos, como por ejemplo los teléfonos celulares, las tablets, el internet, etc. Pero esas destrezas no garantizan un aprendizaje formativo académico, por ello Morales et al. (2015), concluyeron que hay una carencia de formación en el mundo tecnológico en todos los implicados (educandos y docentes), y en consecuencia, hay una demanda sobre formación por las nuevas TIC y una consecutiva combinación en los métodos de enseñanzaaprendizaje.

En este sentido resulta importante la intervención docente para enseñar al estudiante aprovechar en pro del aprendizaje, aquellas herramientas digitales que maneja muy bien. Consecuentemente el docente deberá asumir este reto que hoy por hoy involucra a todos quienes desempeñan la función de educadores debiendo capacitarse en "tres grandes dimensiones: disciplinar, pedagógica y tecnológica, tales componentes deben estar en permanente interacción: conocimiento pedagógico del contenido, cono-cimiento de la utilización de las TIC en los procesos de enseñanza y conocimiento tecnológico, pedagógico y de contenido" (Aguaded y Cabero, 2014, p. 77).

\section{Formación de los profesores universitarios en el uso de las TIC.}

A pesar de que se utilizan las TIC en la educación, existe la inquietud por indagar por qué, si existen tantos recursos tecnológicos para apoyar en el proceso educativo, no todos los docentes los están utilizando. Una de las probables explicaciones es que puede deberse a la falta de conocimiento de cómo se pueden incorporar en el aula; otra explicación es que puede deberse a la poca o inexistente formación que las instituciones ofrecen a sus docentes en el uso pedagógico de estas tecnologías. (Monsiváis et al, 2014 p. 93)

En este contexto aparece también la modalidad híbrida "que mezcla sesiones 
Dany Cristian López-Espinoza; Ángel Enrique Azuero-Azuero

presenciales y en línea, por la calidad de la comunicación tanto cara a cara como indirecta con el uso de los medios de comunicación" (Monsiváis et al., 2014, p. 95). Quizá la utilización de esta modalidad sea en definitiva la alternativa educacional hacia donde conlleva el uso de las herramientas digitales, de allí que la búsqueda de opciones tecnológicas que complementen el trabajo del aula es una inevitable tarea docente, en función de los objetivos propios de cada asignatura.

En el mismo orden de ideas, otro tema que tiene incidencia y que fue analizado por Monsiváis et al. (2014), quienes se refirieron a las estrategias de enseñanza y aprendizaje, al preguntar a los docentes investigados ¿Cómo es un día de clase?, "la mayoría comentó que emplea la técnica expositiva como la más recurrente, lo que puede considerarse como prácticas tradicionales de enseñanza" (p. 98).

Para tener una mayor percepción de lo concluido el artículo presentó la siguiente tabla:

\section{Tabla 1}

Descripción de un día de clase

Descripción de profesores de un día de clase

Profesor 1: Presentación de caso problema, asignar roles a los integrantes, responder en equipo a diferentes preguntas con respecto al caso problema.

Profesor 2: Se inicia el tema con un diagnóstico de lectura previa, hago exposición del tema o los alumnos, y se hacen ejercicios.

Profesor 3: Aplico el tema en imagen y explico los puntos que se verán, provoco el diálogo y razonamiento con los alumnos.

Profesor 4: Expongo la idea, planteo el problema, resolución colectiva, retroalimentación.

Profesor 5: Solicito lectura previa, se elabora el mapa conceptual del tema, se revisa en PowerPoint, se hace un resumen.

Profesor 6: $20 \%$ es teoría de mi parte; $60 \%$, trabajo en equipos, prácticas, salidas de campo; $20 \%$, cuestionarios.

Profesor 7: Podemos iniciar con una presentación por parte de los alumnos, o una pregunta que despierte el interés o bien un tema que introduzca la importancia del tema a tratar.

Profesor 8: Cátedra, diapositivas, exposiciones, preguntas. 
Dany Cristian López-Espinoza; Ángel Enrique Azuero-Azuero

Es evidente que no puede existir un desprendimiento entre educación y tecnología; por lo tanto, el uso de las mismas va convirtiéndose en el equivalente a utilizar pizarra, marcador, texto, lápiz, etc. Incluso se puede decir, que los recursos mencionados de algún modo están inmersos en las herramientas digitales, por ello surge la imperante necesidad de que exista una constante capacitación a los profesores en el uso de las TIC, en base a una educación mediática y pedagógica. "Lograr entornos virtuales de enseñanza-aprendizaje efectivos, es decir, que permitan obtener buenos resultados en términos de construcción de conocimientos, requiere considerar cambios metodológicos y en las estrategias didácticas que allí se despliegan" (Giudicessi et al., 2016, p. 11).

Chan (2016), en referencia a la virtualización de la educación superior; mencionó que esta se plantea como una actualidad en la que se aplican formas educativas cuyos potenciales se ven incrementados con la aplicación de las TIC, que va paralelo con el desarrollo económico y cultural (p. 3), lo cual es otro indicador que incentiva la promulgación de la necesidad de un personal docente preparado para afrontar e involucrarse pedagógicamente con las tendencias tecnológicas como por ejemplo las que han sido seleccionadas a continuación:

Las doce tecnologías elegidas para el horizonte 2013-2018 en América Latina fueron: Para un año o menos: aprendizaje en línea, contenido abierto, entornos colaborativos y redes sociales. Para dos a tres años: analíticas de aprendizaje, aprendizaje móvil, aprendizaje personalizado y realidad aumentada. Para cuatro a cinco años: aprendizaje automático, impresión 3D, internet de las cosas y laboratorios virtuales y remotos. (Chan, 2016, p. 24), siendo pertinente tener en cuenta las nuevas tendencias pedagógicas como el aprendizaje en línea.

Por ello la importancia de cada una, se verá reflejada de acuerdo al contexto y necesidades de enseñanza y aprendizaje. En referencia a lo expuesto, Rama (2012) afirmó que la virtualización es una de las macrotendencias dominantes en la educación superior en los últimos años. Por ello es necesario precisar estudios que aporten a la especialización docente orientada a involucrarse activa y permanentemente en la aplicación de estas tendencias, de tal forma que su posición 
Dany Cristian López-Espinoza; Ángel Enrique Azuero-Azuero

de educador no vaya perdiendo relevancia en esta nueva sociedad del conocimiento, En los últimos años la educación superior en el Ecuador, ha marcado notables transformaciones, que permiten una proyección de mejoramiento de la calidad educativa. Son de conocimiento público acciones gubernamentales como: incremento en la inversión en educación superior, incluso que supera a otros países de América del Sur; y, una Ley Orgánica de Educación Superior que incluye entre otros principios: calidad, pertinencia, integralidad, autodeterminación; la evaluación y categorización que incluso ha llevado al cierre de universidades por la falta de calidad académica y ha servido como factor impulsador para el desarrollo de todas las universidades ecuatorianas. Esto ha derivado en el mejoramiento de la calidad académica de los estudiantes.

\section{METODOLOGÍA}

La investigación se realizó con un enfoque mixto, considerando criterios de Hernández, Fernández y Baptista (2014), al recolectar los datos en un momento único, se planteó un diseño no experimental transeccional para aportar evidencia respecto de los lineamientos de la investigación, es así que se observó el fenómeno investigado tal como se da en su contexto natural, no se tuvo manipulación deliberada de variables. Así también el diseño transeccional fue descriptivo, dado que se indagó la incidencia de las variables planteadas en la población especificada. En cuanto a los métodos la investigación refleja un referente cuantitativo porque se utilizó la recolección de datos para establecer patrones de comportamiento, con base en la medición numérica y el análisis estadístico. Además, se caracteriza porque se planteó un problema de estudio delimitado y concreto en la carrera de Contabilidad y Auditoría de la Universidad Católica de Cuenca, extensión La Troncal durante el periodo abril - agosto 2017. En cuanto a la parte cualitativa utilizó la recolección de datos sin medición numérica para descubrir o afinar preguntas de investigación en el proceso de interpretación.

En cuanto al enfoque cuantitativo el universo fue de 15 docentes, correspondientes a la carrera de Contabilidad y Auditoría de la Universidad Católica de Cuenca, extensión La Troncal para el periodo abril - agosto 2017. El tamaño de la muestra 


\section{CIENCIAMATRIA}

Revista Interdisciplinaria de Humanidades, Educación, Ciencia y Tecnología

Año VI. Vol. VI. N${ }^{\circ}$. Edición Especial. 2020

Hecho el depósito de ley: pp201602FA4721

ISSN-L: 2542-3029; ISSN: 2610-802X

Universidad Nacional Experimental Francisco de Miranda (UNEFM). Santa Ana de Coro. Venezuela

Dany Cristian López-Espinoza; Ángel Enrique Azuero-Azuero

tiene un nivel de confianza del $95 \%$ y un margen de error del $5 \%$. Para el enfoque cualitativo se organizaron las entrevistas respectivas según lo planificado y el correspondiente análisis de documentos. Los docentes se caracterizaron por ser los informantes claves.

Según los 'procedimientos de recolección de la información; los datos cuantitativos conseguidos con las encuestas, fueron procesados mediante métodos estadísticos, matemáticos para el análisis de frecuencias y medidas de resumen de tendencia central y dispersión. Mientras que para el procesamiento de los datos cualitativos se utilizó categorías temáticas y codificación de la información para el análisis del contenido.

\section{RESULTADOS}

Como estaba previsto, durante el periodo abril - agosto del año 2017, se procedió a evaluar las tendencias pedagógicas y las herramientas digitales del aula en la carrera de Contabilidad y Auditoría de la Universidad Católica de Cuenca, extensión La Troncal. Tanto las tendencias en el campo de la educación como el uso de herramientas digitales fueron valoradas a través de instrumentos estadísticos aplicados a los profesores. Como ya se mencionó para los profesores se emplearon: ficha de análisis de los sílabos, ficha de observaciones áulicas y cuestionario de encuesta.

\section{Tendencias Pedagógicas}

A continuación, se presentan los resultados correspondientes a cinco tendencias pedagógicas propuestas en el cuestionario de evaluación aplicado a docentes. 


\section{Tabla 2. Tendencias pedagógicas según docentes}

\begin{tabular}{lccc}
\hline & \multicolumn{3}{c}{ Docentes } \\
\cline { 2 - 4 } Aprendizaje Basado en Retos & $\mathrm{N}$ & $\square$ & $\mathrm{s}$ \\
Aprendizaje Basado en Proyectos & 14 & 3,6 & 0,8 \\
Aprendizaje Invertido & 14 & 4,4 & 1,1 \\
Aprendizaje Vivencial & 14 & 3,4 & 0,9 \\
Aprendizaje Flexible & 14 & 3,6 & 1,3 \\
Otro & 14 & 3,6 & 1,0 \\
Nuevas Tendencias Pedagógicas & 3 & 4,3 & 1,2 \\
\hline
\end{tabular}

Nota. $n=$ muestra de participantes, $\square=$ =media o promedio de la muestra en una escala de mínimo 1 y máximo $5, s$ = desviación estándar que señala cuánto se alejan los datos con respecto a la media.

Los docentes señalaron que la tendencia pedagógica con mayor acogida en su contexto universitario corresponde al Aprendizaje Basado en Proyectos, el cual es utilizado frecuentemente; a este le sigue el que señala otro (es decir algún método de enseñanza diferente a los expuestos), en él, tres docentes sostienen que emplean frecuentemente métodos afines al constructivismo. Entre las opciones seleccionadas, también se advierte al Aprendizaje Basado en Retos, el cual, se evidencia ser utilizado ocasionalmente. Del mismo modo, la tendencia de Aprendizaje Vivencial está siendo usada ocasionalmente y con esta misma equivalencia se encontró a la tendencia pedagógica de Aprendizaje Invertido.

En las observaciones áulicas realizadas a cada docente se evidenció que la mayoría de los catedráticos emplea el método de Aprendizaje Basado en Proyectos. Mientras que, un grupo menor realizan clases magistrales o grupos de trabajo. En cuanto a las tendencias tecnológicas investigadas que más aplican los docentes de la carrera de contabilidad y auditoría, se observó el uso de los Entornos Personalizados de Aprendizaje.

Es importante precisar que todas las tendencias pedagógicas son susceptibles de promediar en la medida que permiten generar una variable general que podría entenderse como Nuevas Tendencias Pedagógicas. 


\section{Herramientas Digitales (tendencias tecnológicas).}

En los cuestionarios aplicados, se consultó la frecuencia de uso de ciertas tecnologías, cuyos resultados se presentan en la siguiente tabla.

Tabla 3. Tendencias tecnológicas según docentes

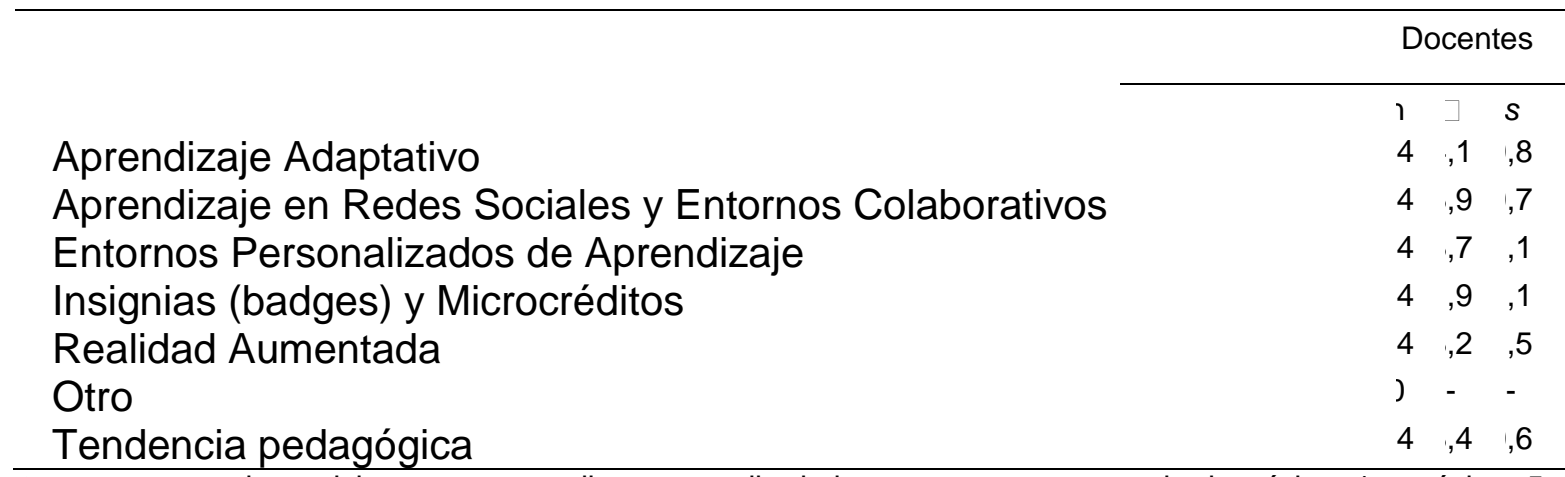

Nota. $n=$ muestra de participantes, $\square=$ media o promedio de la muestra en una escala de mínimo 1 y máximo 5 , $S=$ desviación estándar que señala cuánto se alejan los datos con respecto a la media.

En relación a lo expuesto se puede notar una tendencia a utilizar el Aprendizaje Adaptativo, el mismo que maneja un método computacional para establecer una práctica individual de aprendizaje, como el que mayor frecuencia presenta en el proceso de enseñanza. Una adecuación a las opciones de respuesta originales sería que se usa frecuentemente.

El siguiente aspecto implica al Aprendizaje en Redes Sociales y Entornos Colaborativos el cual tiene también un valor equivalente a "frecuentemente" con tendencia a "ocasionalmente". En menor medida se encuentra Entornos Personalizados de Aprendizaje que permiten configurar a los propios estudiantes la gestión de su aprendizaje. Raramente se emplean Insignias y Microcréditos, lo que supone que no se certifica el aprendizaje informal de los estudiantes.

\section{Correlación entre tendencias pedagógicas y herramientas digitales.}

Se han calculado correlaciones bivariadas de Pearson con significancia bilateral para las tendencias pedagógicas (Columnas) y las tecnológicas, recursos tecnológicos a disposición y uso de dichos recursos a disposición. 
CIENCIAMATRIA

Revista Interdisciplinaria de Humanidades, Educación, Ciencia y Tecnología

Año VI. Vol. VI. Nº 1. Edición Especial. 2020

Hecho el depósito de ley: pp201602FA4721

ISSN-L: 2542-3029; ISSN: 2610-802X

Universidad Nacional Experimental Francisco de Miranda (UNEFM). Santa Ana de Coro. Venezuela

Dany Cristian López-Espinoza; Ángel Enrique Azuero-Azuero

Tabla 4. Correlación de tendencias pedagógicas docentes con tendencias, recursos y uso de recursos tecnológicos

\begin{tabular}{|c|c|c|c|c|c|c|c|}
\hline & & $\begin{array}{l}\text { pr. Basado en } \\
\text { Retos ABR }\end{array}$ & $\begin{array}{l}\text { pr. Basado en } \\
\text { royectos ABP }\end{array}$ & or. Invertido Al & ir. Vivencial AV & or. Flexible AF & $\begin{array}{c}\text { Tendencia } \\
\text { pedagógica }\end{array}$ \\
\hline \multirow{3}{*}{$\begin{array}{l}\text { Aprendizaje } \\
\text { Adaptativo }\end{array}$} & rr. & 0,150 & $-0,377$ & $-0,047$ & $-0,178$ & 0,039 & $-0,121$ \\
\hline & \multirow[t]{2}{*}{ ।. } & 0,610 & 0,184 & 0,874 & 0,543 & 0,894 & 0,680 \\
\hline & & 14 & 14 & 14 & 14 & 14 & 14 \\
\hline \multirow{3}{*}{$\begin{array}{l}\text { Redes Socia y } \\
\text { Entornos } \\
\text { Colaborativos }\end{array}$} & \multirow{3}{*}{$\begin{array}{l}\text { rr. } \\
\text { ।. }\end{array}$} & 0,206 & 0,138 & 0,300 & $-0,112$ & $-0,044$ & 0,191 \\
\hline & & 0,481 & 0,638 & 0,297 & 0,703 & 0,880 & 0,512 \\
\hline & & 14 & 14 & 14 & 14 & 14 & 14 \\
\hline Entornos & rr. & 0,305 & $-0,151$ & 0,145 & $-0,253$ & $-0,192$ & $-0,137$ \\
\hline \multirow{2}{*}{$\begin{array}{l}\text { Personalizados } \\
\text { de Aprendizaje }\end{array}$} & \multirow[t]{2}{*}{ ।. } & 0,289 & 0,606 & 0,621 & 0,383 & 0,510 & 0,640 \\
\hline & & 14 & 14 & 14 & 14 & 14 & 14 \\
\hline \multirow{2}{*}{$\begin{array}{l}\text { Insignias y } \\
\text { Microcréditos }\end{array}$} & rr. & 0,024 & 0,119 & 0,070 & 0,424 & 0,148 & 0,250 \\
\hline & I. & $\begin{array}{c}0,936 \\
14\end{array}$ & $\begin{array}{c}0,685 \\
14\end{array}$ & $\begin{array}{c}0,811 \\
14\end{array}$ & $\begin{array}{c}0,130 \\
14\end{array}$ & $\begin{array}{c}0,615 \\
14\end{array}$ & $\begin{array}{c}0,388 \\
14\end{array}$ \\
\hline \multirow{2}{*}{$\begin{array}{l}\text { Realidad } \\
\text { Aumentada }\end{array}$} & rr. & $0,623^{\pi}$ & $-0,109$ & 0,349 & 0,399 & 0,220 & $0,539^{\pi}$ \\
\hline & ।. & $\begin{array}{c}0,017 \\
14\end{array}$ & $\begin{array}{c}0,710 \\
14\end{array}$ & $\begin{array}{c}0,221 \\
14\end{array}$ & $\begin{array}{c}0,158 \\
14\end{array}$ & $\begin{array}{c}0,451 \\
14\end{array}$ & $\begin{array}{c}0,047 \\
14\end{array}$ \\
\hline \multirow{3}{*}{$\begin{array}{l}\text { Uso de herr. } \\
\text { digitales en el } \\
\text { aula }\end{array}$} & rr. & $0,562^{\pi}$ & $-0,147$ & 0,338 & 0,202 & 0,102 & 0,350 \\
\hline & \multirow[t]{2}{*}{ ।. } & 0,036 & 0,617 & 0,238 & 0,488 & 0,728 & 0,219 \\
\hline & & 14 & 14 & 14 & 14 & 14 & 14 \\
\hline \multirow{4}{*}{$\begin{array}{l}\text { Lab. de } \\
\text { computación } \\
\text { internet }\end{array}$} & rr. & 0,323 & $-0,028$ & 0,462 & $-0,135$ & $-0,387$ & 0,014 \\
\hline & \multirow[t]{2}{*}{ I. } & 0,259 & 0,925 & 0,096 & 0,645 & 0,172 & 0,963 \\
\hline & & 14 & 14 & 14 & 14 & 14 & 14 \\
\hline & rr. & $-0,122$ & 0,113 & 0,483 & $0,551^{\star}$ & 0,162 & 0,474 \\
\hline Proyectores & ।. & $\begin{array}{c}0,678 \\
14\end{array}$ & $\begin{array}{c}0,700 \\
14\end{array}$ & $\begin{array}{c}0,080 \\
14\end{array}$ & $\begin{array}{c}0,041 \\
14\end{array}$ & $\begin{array}{c}0,580 \\
14\end{array}$ & $\begin{array}{c}0,087 \\
14\end{array}$ \\
\hline \multirow{3}{*}{$\begin{array}{l}\text { Pizarras } \\
\text { digitales }\end{array}$} & rr. & 0,411 & 0,156 & 0,307 & 0,078 & 0,013 & 0,272 \\
\hline & \multirow[t]{2}{*}{ ।. } & 0,144 & 0,595 & 0,286 & 0,792 & 0,965 & 0,346 \\
\hline & & 14 & 14 & 14 & 14 & 14 & 14 \\
\hline \multirow{2}{*}{$\begin{array}{l}\text { Plataformas } \\
\text { educativas }\end{array}$} & rr. & 0,220 & $-0,151$ & 0,145 & $-0,092$ & $-0,405$ & $-0,194$ \\
\hline & I. & 0,450 & 0,606 & 0,621 & 0,755 & 0,151 & 0,505 \\
\hline & rr. & 0,419 & $\begin{array}{l}14 \\
0,079\end{array}$ & $\begin{array}{l}14 \\
0,101\end{array}$ & $\begin{array}{c}14 \\
-0,120\end{array}$ & $\begin{array}{c}14 \\
-0,011\end{array}$ & $\begin{array}{l}14 \\
0,076\end{array}$ \\
\hline $\begin{array}{l}\text { Software de } \\
\text { aplicación }\end{array}$ & 1. & $\begin{array}{c}0,136 \\
14\end{array}$ & $\begin{array}{c}0,789 \\
14\end{array}$ & $\begin{array}{c}0,732 \\
14\end{array}$ & $\begin{array}{c}0,684 \\
14\end{array}$ & $\begin{array}{c}0,972 \\
14\end{array}$ & $\begin{array}{c}0,797 \\
14\end{array}$ \\
\hline & rr. & 0,510 & 0,106 & 0,386 & 0,009 & $-0,114$ & 0,214 \\
\hline Recursos & I. & 0,063 & 0,718 & 0,173 & 0,975 & 0,698 & 0,462 \\
\hline tecnologicos & & 14 & 14 & 14 & 14 & 14 & 14 \\
\hline Lab. de & rr. & 0,162 & $-0,172$ & 0,479 & 0,139 & $-0,167$ & 0,139 \\
\hline computación & ।. & 0,581 & 0,557 & 0,083 & 0,635 & 0,568 & 0,636 \\
\hline internet & & 14 & 14 & 14 & 14 & 14 & 14 \\
\hline & rr. & 0,220 & $-0,151$ & 0,145 & 0,337 & 0,162 & 0,278 \\
\hline Proyectores & I. & 0,450 & 0,606 & 0,621 & 0,239 & 0,580 & 0,335 \\
\hline & & 14 & 14 & 14 & 14 & 14 & 14 \\
\hline & rr. & 0,411 & 0,156 & 0,307 & 0,078 & 0,013 & 0,272 \\
\hline Pizarras digitales & I. & 0,144 & 0,595 & 0,286 & 0,792 & 0,965 & 0,346 \\
\hline & & 14 & 14 & 14 & 14 & 14 & 14 \\
\hline & rr. & $-0,122$ & 0,113 & $-0,193$ & $-0,092$ & 0,162 & $-0,015$ \\
\hline Plataformas & ।. & 0,678 & 0,700 & 0,508 & 0,755 & 0,580 & 0,959 \\
\hline & & 14 & 14 & 14 & 14 & 14 & 14 \\
\hline & rr. & 0,316 & $-0,085$ & 0,082 & $-0,052$ & $-0,068$ & 0,010 \\
\hline $\begin{array}{l}\text { Sottware de } \\
\text { aplicación }\end{array}$ & ।. & 0,271 & 0,773 & 0,782 & 0,861 & 0,816 & 0,973 \\
\hline & & 14 & 14 & 14 & 14 & 14 & 14 \\
\hline & rr. & $-0,444$ & $-0,284$ & 0,061 & $-0,038$ & $-0,051$ & $-0,213$ \\
\hline Wifi libre acceso & I. & 0,111 & 0,325 & 0,837 & 0,896 & 0,863 & 0,465 \\
\hline & & 14 & 14 & 14 & 14 & 14 & 14 \\
\hline & rr. & 0,316 & $-0,103$ & 0,354 & 0,084 & $-0,052$ & 0,166 \\
\hline Uso de & ।. & 0,271 & 0,726 & 0,214 & 0,776 & 0,859 & 0,571 \\
\hline & & 14 & 14 & 14 & 14 & 14 & 14 \\
\hline
\end{tabular}

* La correlación es significativa en el nivel 0,05 (2 colas)

** La correlación es significativa en el nivel 0,01 ( 2 colas). 
Dany Cristian López-Espinoza; Ángel Enrique Azuero-Azuero

Se encontró asociación entre la tendencia pedagógica denominada Aprendizaje Basado en Retos y la tendencia tecnológica de la realidad aumentada, la asociación reportada es de un $62,3 \%$, la regresión lineal de esta correlación ofrece una explicación de una variable sobre la otra del $38,82 \%$. En efecto, incluso la realidad aumentada tiene asociación con el promedio de tendencias pedagógicas en general con una correlación $53,9 \%$ y una regresión de $31,62 \%$.

En lo que se refiere a las entrevistas realizadas, se obtuvieron 2 de las 3 planificadas para el proceso. La primera se realizó a la Subdirectora de Carrera de Contabilidad y Auditoría, a quien se le preguntó si los docentes de la carrera aprovechan los recursos tecnológicos disponibles y de ser así, ¿de qué manera lo hacen? A lo cual respondió:

"Si... los docentes de la carrera de contabilidad y auditoría, al momento de impartir sus cátedras utilizan las herramientas tecnológicas que dispone cada una de nuestras aulas. Además, contamos con una plataforma de aula virtual, donde el docente puede interactuar con los estudiantes a través de foros, tareas, pruebas en línea.".

Así también se le consultó, si en calidad de responsable académica de la carrera ha podido evidenciar que los docentes planifican haciendo uso de las herramientas digitales en el aula; y en este sentido la entrevistada afirmó que la universidad cuenta un sistema ERP (Enterprise Resource Planning), en donde se registran los sílabos y las planificaciones de cada una de las asignaturas que los docentes imparten. Respecto a las técnicas, métodos o tendencias pedagógicas más utilizadas por los docentes de la carrera, la Ing. Ramón mencionó que la universidad: "Utiliza la pedagogía critica, que consiste en el desarrollo de la personalidad y capacidades cognitivas, potencia el trabajo grupal, formando a un estudiante crítico, participativo y reflexivo; dentro de los métodos aplicados por los docentes de la carrera están, solución de problemas, inductivo-deductivo, analítico, y las técnicas que emplean, son talleres, casos de estudios, lluvia de ideas, etc.".

Se le preguntó a la entrevistada si consideraba importante el fortalecimiento y la actualización del personal docente en cuanto a la práctica de las tendencias 
CIENCIAMATRIA

Revista Interdisciplinaria de Humanidades, Educación, Ciencia y Tecnología

Año VI. Vol. VI. N${ }^{\circ}$. Edición Especial. 2020

Hecho el depósito de ley: pp201602FA4721

ISSN-L: 2542-3029; ISSN: 2610-802X

Universidad Nacional Experimental Francisco de Miranda (UNEFM). Santa Ana de Coro. Venezuela

Dany Cristian López-Espinoza; Ángel Enrique Azuero-Azuero

pedagógicas y el uso adecuado de las herramientas digitales. Con una respuesta afirmativa mencionó que:

"La formación permanente es un proceso necesario para todas y todos los docentes porque permite su actualización de acuerdo a los avances de la pedagogía, los mismos que deben estar en relación con el modelo educativo que se plantee y garantice la innovación que el sistema educativo requiere, así mismo el docente tiene que estar actualizado en el uso de las herramientas digitales con el objetivo que pueda ofrecer diversas formas didácticas y metodológicas que proporcionen la creatividad en los estudiantes".

En este sentido también señaló que la universidad evalúa cada ciclo a los docentes y en función de las calificaciones que obtengan, se dispone la obligatoriedad para asistir a cursos de pedagogía.

Finalmente, la Subdirectora de carrera indicó que, dentro del programa de capacitación docente de la universidad, se brinda actualización en el uso de las TIC y pedagogía, a todos los catedráticos de la extensión universitaria.

Una segunda entrevista se realizó al Responsable de laboratorios y área de cómputo, quien sostuvo también el hecho de que los docentes si aprovechan los recursos tecnológicos a disposición, agregando que la formación profesional y experiencia de quienes se desempeñan como catedráticos, está vinculada directamente con el manejo de recursos tecnológicos.

Explicó que entre los recursos que el alumno utiliza en lo relacionado a las tecnologías son, el uso del internet, en donde cada estudiante tiene su usuario y contraseña, además mencionó la utilización del EVA (Entorno Virtual de Aprendizaje) que permite la interacción profesor - estudiante; las bases de datos científicas y las bibliotecas virtuales.

En lo que se refiere a la implementación de recursos tecnológicos, el entrevistado consideró que sería importante la adquisición de pizarras digitales, en virtud de que la universidad no dispone de ellas, así también explicó que hay falta de equipos de audio en cada aula. Sin embargo, aclaró que la universidad cuenta con laboratorios debidamente equipados y funcionales, pero que lo ideal sería disponer de aulas con 
Dany Cristian López-Espinoza; Ángel Enrique Azuero-Azuero

mejor dotación de equipos tecnológicos, para una mayor comodidad de todos los estudiantes.

En el tema de cuáles son las técnicas, métodos o tendencias pedagógicas más utilizadas por profesores de la carrera investigada, él entrevistado afirmó que lo más utilizado son las aulas virtuales, el uso de imágenes en proyección, videos y además el sistema ERP que permite subir las notas, control de asistencia etc.

En la consulta respecto a la importancia del fortalecimiento y la actualización del personal docente en cuanto a la práctica de las tendencias pedagógicas y el uso adecuado de las herramientas digitales, el entrevistado indicó:

"Siempre es importante estar a la vanguardia de las tecnologías hoy en día, porque si nos fijamos para todo ahora necesitamos de la tecnología y mucho más si se trata de una entidad educativa a la cual debemos tener siempre tanto en equipos, como en software las actualizaciones de última, para así brindar una mejor enseñanza y aprendizaje al estudiante".

Finalmente, el entrevistado agregó que cuentan con correo institucional netamente de la universidad el mismo que sirve como enlace principal para el envío-recepción de información, precisó que dentro del mismo servicio emplean el paquete de Microsoft Office en línea y que todo esto implica el manejo constante de la Web 2.0 por parte de los miembros de la comunidad universitaria.

\section{CONCLUSIONES}

Algunas conclusiones importantes sobre los resultados de la investigación son las siguientes:

La tendencia pedagógica con mayor acogida en la universidad investigada corresponde al Aprendizaje Basado en Proyectos que es utilizado frecuentemente y con un $57,1 \%$ en las clases observadas. Con una menor intensidad, pero utilizado también de manera frecuente está el Aprendizaje Flexible. Esto implica que las mencionadas tendencias han contribuido a fortalecer la enseñanza.

No se constató el uso frecuente de otras tendencias pedagógicas analizadas en este proceso.

Las tendencias tecnológicas que se utiliza con mayor frecuencia son: los Entornos 
Dany Cristian López-Espinoza; Ángel Enrique Azuero-Azuero

Personalizados de Aprendizaje de los cuales se observó una utilización del $71,4 \%$ de los docentes; el Aprendizaje Adaptativo y el Aprendizaje en Redes Sociales y Entornos Colaborativos también son utilizados frecuentemente. El uso de otras tendencias tecnológicas del grupo de investigadas, no se da con mayor frecuencia. Es así que las herramientas digitales de uso frecuente, son recursos que permiten la construcción significativa del conocimiento.

Los recursos tecnológicos más empleados en el proceso de enseñanza aprendizaje son: proyectores, laboratorios, plataformas educativas y wifi; aunque en este último se observó que no es frecuente el uso de internet en las aulas por parte de los docentes.

En la planificación de la metodología en los sílabos, no se observó un nivel importante de inclusión de las tendencias pedagógicas y tecnológicas estudiadas.

Si se incrementa el uso de las tendencias pedagógicas, entonces se hace necesario aumentar el uso de las herramientas digitales en el aula.

Es importante el fortalecimiento y la actualización del personal docente en cuanto a la práctica de nuevas tendencias pedagógicas y el uso adecuado de las herramientas digitales en el aula.

\section{REFERENCIAS CONSULTADAS}

1. Abrigo-Córdova, I., Granados Gómez, D., Sánchez Sulú, N., \& Celi Vivanco, Y. (2019). El aula virtual: una experiencia educativa desde diversos ámbitos universitarios latinoamericanos. CIENCIAMATRIA, 6(10), 359-385. https://doi.org/10.35381/cm.v6i10.136

2. Aguaded, I., Cabero, J. (2014). Avances y retos en la promoción de la innovación didáctica con las tecnologías emergentes e interactivas. Educar Especial $30^{\circ}$ aniversario, 67-83. doi: http://dx.doi.org/10.5565/rev/educar.691

3. Chan, M. (2016). La virtualización de la educación superior en América Latina: entre tendencias y paradigmas. RED-Revista de Educación a Distancia 48(1), 1-32. 30-Ene-2016. Recuperado de http://www.um.es/ead/red/48/chan.pdf

4. Fainholc, B., Nervi, H., Romero, R. y Halal, C. (2013). La formación del profesorado y el uso pedagógico de las TIC. Revista de Educación a Distancia, $\quad N^{\circ} \quad 38 . \quad$ Recuperado de http://revistas.um.es/red/article/view/234081/0 
Dany Cristian López-Espinoza; Ángel Enrique Azuero-Azuero

5. García Morales, A., \& González Ten Meer, C. (2018). La investigación acción participativa (IAP), como herramienta para identificar la gestión de la calidad docente. EPISTEME KOINONIA, 1(1), 3-17. Recuperado de https://fundacionkoinonia.com.ve/ojs/index.php/epistemekoinonia/article/view/ $\underline{487 / 651}$

6. Giudicessi, S., Martínez, M., Saavedra, S., Cascone, O. y Camperi, S. (2016). Las Tecnologías y la Enseñanza en la Educación Superior. Un Simulador Aplicado a la Integración de Conceptos Enseñados en Cursos de Posgrado. Revista Iberoamericana de Evaluación Educativa, 9(2), 9-28. Recuperado de http://revistainformaticaeducativa.unan.edu.ni/index.php/ie/article/view/24

7. Hernández, C., Ayala-García, E., Gamboa, A. (2015). Modelo de competencias TIC para docentes. Una propuesta para la construcción de contextos educativos innovadores y la consolidación de aprendizajes en educación superior. Katharsis: Revista de Ciencias Sociales, № 22, 221-265. Recuperado de https://dialnet.unirioja.es/servlet/articulo?codigo=5796593

8. Hernández, R., Fernández, C. y Baptista, M. (2010). Metodología de la Investigación. México, México: McGraw-Hill / Interamericana Editores, S.A. de C.V.

9. Manzanilla, H., Dorantes, M., y Cordero, M. (2016). Internacionalización de la educación y Tecnologías de la Información y la Comunicación. Un vínculo necesario. Rev. educ. super. sur glob - RESUR №2 jul. dic. 2016. 4-22. Recuperado

http://www.iusur.edu.uy/publicaciones/index.php/RESUR/article/view/20

10. Lattá Arias, C. (2019). Uso de las TIC para proyectos productivos en las instituciones educativas del Municipio Zona Bananera. Magdalena. Colombia. Revista Arbitrada Interdisciplinaria Koinonía, 4(7), 233-246. doi:http://dx.doi.org/10.35381/r.k.v4i7.202

11. Monsiváis, M.I., McAnally, L. y Lavigne, G. (2014). Aplicación y validación de un modelo tecnopedagó $q$ gico de formación docente mediante una plataforma educativa virtual. Revista de Universidad y Socie $\neg$ dad del Conocimiento (RUSC). Vol. 11, n.o 1, 91-107. doi http://dx.doi.org/10.7238/rusc.v11i1.1743

12. Morales, M., Trujillo, J.M. \& Raso, F. (2015). Percepciones acerca de la integración de las TIC en el proceso de enseñanza-aprendizaje de la universidad. Revista de Medios y Educación, 46, 103-117. doi: http://dx.doi.org/10.12795/pixelbit.2015.i46.07. 
13. Observatorio de Innovación Educativa (2016). Reporte Edu Trends: Radar de Innovación Educativa de Preparatoria 2016. Recuperado de https://observatorio.itesm.mx/edutrendsradarpreparatoria2016

14. Pérez, D., Betancourt, E., Silveira, Y., Loza C. (2016). Pedagogía o Educación Afectiva: Necesidad Impostergable en Llas Universidades Ecuatorianas. Investigación y Saberes, Vol. V No. Especial (2016): 34-41. Recuperado de http://utelvt.edu.ec/ojs/index.php/is/article/view/131

15. Rama, C. (2012). La reforma de la virtualización de la Universidad. Guadalajara: UDGVIRTUAL.

16. Sandí, J. y Cruz, M. (2016). Propuesta metodológica de enseñanza y aprendizaje para innovar la educación superior. InterSedes, 17(36), 1-38. Recuperado de http://dx.doi.org/10.15517/isucr.v17i36.27100

17. Vicedo Tomey, Agustín. (2015). ¿Quién debe enseñar qué cosa en educación médica? El papel del profesor y el conocimiento pedagógico del contenido. Educación Médica Superior, 29(3) Recuperado en 14 de marzo de 2017, de http://scielo.sld.cu/scielo.php?script=sci arttext\&pid=S0864214120150003000

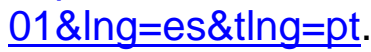

\section{REFERENCES CONSULTED}

1. Abrigo-Córdova, I., Granados Gómez, D., Sánchez Sulú, N., \& Celi Vivanco, Y. (2019). The virtual classroom: an educational experience from various Latin American university fields. SCIENCE, 6 (10), 359-385. https://doi.org/10.35381/cm.v6i10.136

2. Aguaded, I., Cabero, J. (2014). Advances and challenges in the promotion of didactic innovation with emerging and interactive technologies. Educate Special 30th anniversary, 67-83. doi: http://dx.doi.org/10.5565/rev/educar.691

3. Chan, M. (2016). The virtualization of higher education in Latin America: between trends and paradigms. RED-Distance Education Magazine 48 (1), 132. 30-Jan-2016. Recovered from http://www.um.es/ead/red/48/chan.pdf

4. Fainholc, B., Nervi, H., Romero, R. and Halal, C. (2013). Teacher training and pedagogical use of ICT. Distance Education Magazine, No. 38. Retrieved from http://revistas.um.es/red/article/view/234081/0

5. García Morales, A., \& González Ten Meer, C. (2018). Participatory action research (IAP), as a tool to identify teacher quality management. EPISTEME KOINONIA, 
https://fundacionkoinonia.com.ve/ojs/index.php/epistemekoinonia/article/view/ $\underline{487 / 651}$

6. Giudicessi, S., Martínez, M., Saavedra, S., Cascone, O. and Camperi, S. (2016). Technologies and Teaching in Higher Education. A Simulator Applied to the Integration of Concepts Taught in Postgraduate Courses. Iberoamerican Journal of Educational Evaluation, 9 (2), 9-28. Recovered from http://revistainformaticaeducativa.unan.edu.ni/index.php/ie/article/view/24

7. Hernández, C., Ayala-García, E., Gamboa, A. (2015). ICT skills model for teachers. A proposal for the construction of innovative educational contexts and the consolidation of learning in higher education. Katharsis: Journal of Social Sciences, No. 22, 221-265. Recovered from https://dialnet.unirioja.es/servlet/articulo?codigo $=5796593$

8. Hernández, R., Fernández, C. and Baptista, M. (2010). Investigation methodology. Mexico, Mexico: McGraw-Hill / Interamericana Editores, S.A. from C.V.

9. Manzanilla, H., Dorantes, M., and Cordero, M. (2016). Internationalization of education and Information and Communication Technologies. A necessary link. Rev. educ. super. sur glob - RESUR №2 jul. Dec. 2016. 4-22. Recovered from http://www.iusur.edu.uy/publicaciones/index.php/RESUR/article/view/20

10. Lattá Arias, C. (2019). Use of ICT for productive projects in the educational institutions of the Municipality of Banana Zone. Cupcake Colombia. Interdisciplinary Arbitrated Review Koinonía, 4 (7), 233-246. doi: http: //dx.doi.org/10.35381/r.k.v4i7.202

11. Monsiváis, M.I., McAnally, L. and Lavigne, G. (2014). Application and validation of a techno-pedagogical model of teacher training through a virtual educational platform. University and Knowledge Society Magazine (RUSC). Vol. 11, No. 1, 91-107. doi http://dx.doi.org/10.7238/rusc.v11i1.1743

12. Morales, M., Trujillo, J.M. \& Raso, F. (2015). Perceptions about the integration of ICT in the teaching-learning process of the university. Media and Education Magazine, 46, 103-117. doi: http://dx.doi.org/10.12795/pixelbit.2015.i46.07.

13. Observatory of Educational Innovation (2016). Edu Trends Report: High School Educational Innovation Radar 2016. Retrieved from https://observatorio.itesm.mx/edutrendsradarpreparatoria2016

14.Pérez, D., Betancourt, E., Silveira, Y., Loza C. (2016). Pedagogy or Affective Education: Impossible Need in Ecuadorian Universities. Research and Knowledge, Vol. V Special No. (2016): 34-41. Recovered from 


\section{CIENCIAMATRIA}

Revista Interdisciplinaria de Humanidades, Educación, Ciencia y Tecnología

Año VI. Vol. VI. N${ }^{\circ}$. Edición Especial. 2020

Hecho el depósito de ley: pp201602FA4721

ISSN-L: 2542-3029; ISSN: 2610-802X

Universidad Nacional Experimental Francisco de Miranda (UNEFM). Santa Ana de Coro. Venezuela

Dany Cristian López-Espinoza; Ángel Enrique Azuero-Azuero

http://utelvt.edu.ec/ojs/index.php/is/article/view/131

15. Rama, C. (2012). The virtualization reform of the University. Guadalajara: UDGVIRTUAL.

16. Sandí, J. and Cruz, M. (2016). Methodological proposal of teaching and learning to innovate higher education. InterSedes, 17 (36), 1-38. Retrieved from http://dx.doi.org/10.15517/isucr.v17i36.27100

17. Vicedo Tomey, Agustin. (2015). Who should teach what in medical education? The role of the teacher and the pedagogical knowledge of the content. Higher Medical Education, 29 (3) Retrieved on March 14, 2017, from http://scielo.sld.cu/scielo.php?script=sci arttext\&pid=S0864214120150003000

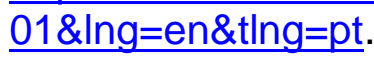

